$2012-5$

\title{
Relative Navigation and Control of a Hexacopter
}

Timothy McLain

Mechanical Engineering Department, Brigham Young University, mclain@byu.edu

Randal W. Beard

Department of Electrical and Computer Engineering, Brigham Young University, beard@ee.byu.edu

Robert C. Leishman

US AFRL Sensors Directorate, Wright-Patterson AFB

John MacDonald

Department of Electrical Engineering, Brigham Young University

Follow this and additional works at: https://scholarsarchive.byu.edu/facpub

Part of the Mechanical Engineering Commons

\section{Original Publication Citation}

Leishman, R., Macdonald, J., McLain, T. and Beard, R. Relative Navigation and Control of a Hexacopter, Proceedings of the IEEE International Conference on Robotics and Automation, pp. 4937-4942, May 2012, St. Paul, Minnesota.

\section{BYU ScholarsArchive Citation}

McLain, Timothy; Beard, Randal W.; Leishman, Robert C.; and MacDonald, John, "Relative Navigation and Control of a Hexacopter" (2012). Faculty Publications. 1947.

https://scholarsarchive.byu.edu/facpub/1947 


\title{
Relative Navigation and Control of a Hexacopter
}

\author{
Robert Leishman, John Macdonald, Tim McLain, Randy Beard
}

\begin{abstract}
This paper discusses the progress made on developing a multi-rotor helicopter equipped with a vision-based ability to navigate through an a priori unknown, GPS-denied environment. We highlight the backbone of our system, the relative estimation and control. We depart from the common practice of using a globally referenced map, preferring instead to keep the position and yaw states in the EKF relative to the current map node. This relative navigation approach allows simple application of sensor updates, natural characterization of the transformation between map nodes, and the potential to generate a globally consistent map when desired. The EKF fuses view matching data from a Microsoft Kinect with more frequent IMU data to provide state estimates at rates high enough to control the vehicles fast dynamics. Although an EKF is used, a nodes and edges graph represents the map. Hardware results showing the quality of the estimates and flights with estimates in the loop are provided.
\end{abstract}

\section{INTRODUCTION}

Vision-based navigation and mapping in complex and unknown GPS-denied environments is an active area of research. Many implementations exist and new ones continue to be developed. However, a majority of the current research is implemented on ground vehicles without including control. A few implementations on quadrotors using laser scanners have been completed [1], [2] but strong assumptions about the nature of the environment are required. We are primarily interested in implementing vision-based navigation in control feedback for aerial vehicles. Vision provides a more rich representation of the vehicle's surroundings, but this comes with the cost of increased processing. Small aerial vehicles also present challenging constraints such as stringent payload limitations and fast vehicle dynamics.

Ahrens, et al. [3] use a single camera to map and navigate a quadrotor vehicle through an unknown environment. An Extended Kalman Filter (EKF) SLAM algorithm estimates global states of the vehicle and visual landmarks.

Blosch, et al. [4] use a downward-looking monocular camera and a graph-based SLAM algorithm to navigate a quadrotor. The vehicle must hover at each waypoint to allow the SLAM algorithm to converge. But estimates are used in the control loop.

R. Leishman is a PhD Candidate, Dept. of Mechanical Engineering, Brigham Young University, Provo, UT 84604 rleish@gmail. com

J. Macdonald is a PhD Candidate, Dept. of Electrical Engineering, Brigham Young University, Provo, UT 84604 johnatbyu@hotmail.com

T. McLain is a Professor and Department Chair, Dept. of Mechanical Engineering, Brigham Young University, Provo, UT 84604 mclain@byu.edu

R. Beard is a Professor, Dept. of Electrical Engineering, Brigham Young University, Provo, UT 84604 beard@byu.edu
Garca Carrillo, et al. [5] implement a stereo visual odometry algorithm and combine the data with an IMU and altimeter in a Kalman filter on a quadrotor. The authors provide results on manual flights and on an autonomous hover flight, however the global estimates are not accompanied by truth information.

Voigt, et al. [6] present a global estimation method that also uses an EKF to combine stereo visual odometry and IMU information. To make the relative visual odometry update global, additional variables that account for the position and orientation (pose) of the last image are added to the state.

Most current implementations utilize global states for the estimation and control of rotorcraft. Requiring global states incurs difficulties such as the need for additional states to incorporate measurements, waiting periods for global consistency, and schemes to accommodate large jumps in pose when loop closures occur. In contrast, we seek to develop a vision-based aerial platform with the ability to navigate using relative states and a graph-based map through an a priori unknown, GPS-denied environment. We base our approach on the work of Konolige, et al. in [7]. Although they implement their algorithm on ground robots and do not present results with the estimation in the control feedback, they provide several features we seek to utilize. The key points are the view matching, nodes and edges graph, place recognition, and optimization algorithms.

A graph-based map is constructed in [7] as the vehicle compares its current stereo image pair to a reference view (called a 'keyframe') using the view matching. Once the overlap between the current view and the keyframe becomes too small, a new keyframe is declared and navigation continues relative to that reference. The graph consists of the nodes, saved keyframes each with an associated pose, and the edges, estimated transformations between those poses. Drift in global position estimates in [7] are constrained by using visual place recognition to identify when the vehicle has returned to a previous portion of the map. A nonlinear optimization routine [8] determines the most likely global arrangement of the nodes given the edge constraints.

This approach is attractive for a number of reasons. It scales well to large environments. Real-time navigation is relative to the current keyframe. Thus the place recognition and nonlinear optimization can run opportunistically without impacting the current relative estimation and control. Also, the saved keyframes represent a rich source of information for path planning and other higher-lever tasks.

We are in the process of adapting [7] to our application. An Extended Kalman Filter (EKF) performs the navigation 
and a nodes-and-edges graph comprises the map.

This paper present the initial results achieved in implementing this mapping and navigation method. An explaination of the relative navigation is in Section II. Section III introduces the system architecture. Section IV provides the results of the current system. Conclusions and future work are in Section V.

\section{Relative NaVigation}

In a departure from what is common practice, we use position and yaw states that are relative to the current node in the map. Typically, the states maintained in the EKF are with respect to the inertial frame. The illustrated map in Figure 1 shows the relative approach. A vector chain of nodes and edges connect the hexacopter to the inertial frame. Global position for the vehicle can be retrieved by summing the edges and the current state, when expressed in the same coordinate frame. At each node there is a local reference frame. The position and yaw angle of the vehicle are relative to that local reference frame.

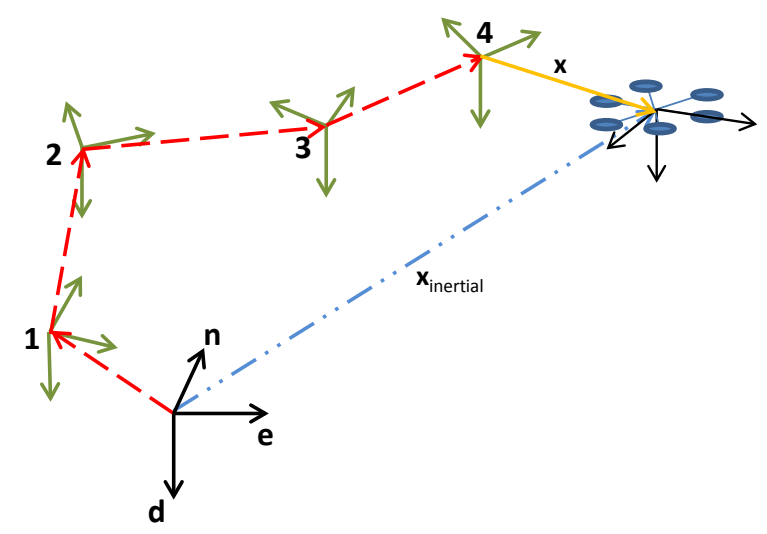

Fig. 1. Relative navigation through nodes and edges. As the vehicle flies through the environment, nodes and edges are created. The vehicle state is relative to the most current node, node four in this example. The inertial state of the vehicle can be found, if necessary, through summing the edges in the map and the current state.

There are several advantages that accompany a relative navigation approach. Ease of updating states with sensors, easy definition of edge mean and covariance information and the flexibility of using a globally consistent map when desired are among the most beneficial.

Exteroceptive sensors provide relative information. In particular, view matching provides the change in 6DOF pose between two images taken at different locations. By comparing the current image with the node keyframe and expressing the result in the current node frame, the position and yaw are updated directly. This simplification eliminates additional required states or registration of images against a set of images to arrive at global updates.

Defining edges between two nodes is a simple matter of saving the relative portions of the state and covariance just before a new node is created. The covariance can be used to compute an understanding of the confidence of the current global position.

This relative approach is also valid with and without loop closure constraints. The local navigation in the EKF takes place regardless of global changes within the map, making it a more flexible approach. Without loop closure it is clear that the map will drift and not remain globally consistent. However, the relative relationship between nodes maintains a consistent topological and locally metric relationship between saved locations. Therefore, the map can be traversed, even back to the starting location, by using these relative relationships.

When loop closure constraints are included, from a visual place-recognition algorithm for example, the map can be kept globally consistent through optimization methods. When loops are closed, it is common for large jumps in the global location to occur. As the vehicle navigates with respect to a local node, global optimization can proceed as a background process, continually making changes without causing harm to the estimation and navigation.

\section{A. Node Frame}

We choose to express the node frame in a different coordinate frame than the reference image frame of the keyframe. The node frame is defined by a coordinate frame where the $\mathbf{d}_{j}$ direction is parallel to the inertial $\mathbf{d}$ direction and the $\mathbf{f}_{j}$ axis is defined by the projection of the body $\mathbf{b}_{\mathbf{x}}$ axis onto the global $\mathbf{n}$-e plane, at the instant the image is taken. The $\mathbf{s}_{j}$ axis is defined to make a right-hand coordinate system. This setup preserves the heading of the vehicle and maintains a global understanding of which direction is down, which keeps the roll and pitch angles defined correctly relative to the global n-e plane.

\section{SyStem ARCHITECTURE}

The system architecture is illustrated by the diagram in Figure 2. We used a similar setup in previous work [9]. In this section we describe the current implementation of each major block in Figure 2.

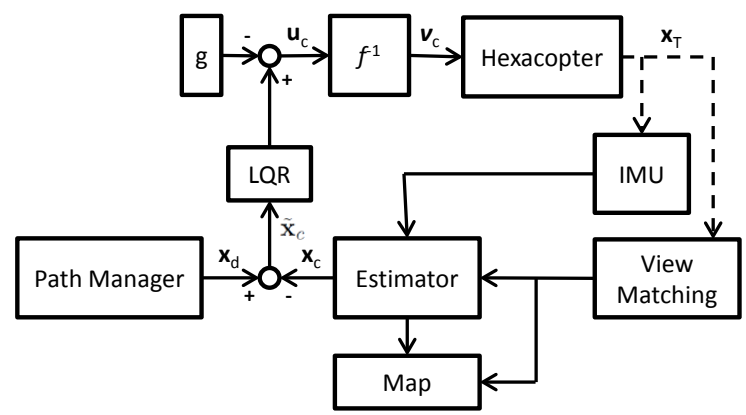

Fig. 2. System diagram for the relative navigation hexacopter. 


\section{A. Hexacopter Model}

The states we estimate are with respect to the current node, node $j$,

$$
\hat{\mathbf{x}}=\left[\begin{array}{llllllllllll}
\hat{f}_{j} & \hat{s}_{j} & \hat{d}_{j} & \hat{\psi}_{j} & \hat{\phi} & \hat{\theta} & \hat{u} & \hat{v} & \hat{w} & \hat{\beta}_{x} & \hat{\beta}_{y} & \hat{\beta}_{z}
\end{array}\right]^{\top} .
$$

The positions $\hat{f}_{j}, \hat{s}_{j}$ and $\hat{\mathbf{d}}_{j}$ are the displacements with respect to node $j$. The yaw angle $\hat{\psi}_{j}$ is a positive, righthanded rotation about the $\mathbf{d}_{j}$ axis. The other Euler angles, $\hat{\phi}$ and $\hat{\theta}$, are the roll and pitch angles respectively. The bodyfixed frame velocities are $\hat{u}, \hat{v}$ and $\hat{w}$. We also estimate the gyroscope biases $\hat{\beta}_{x}, \hat{\beta}_{y}$ and $\hat{\beta}_{z}$. We assume that the accelerometer biases change slowly enough to be removed through calibration.

The inputs to the model are simply the gyroscope measurements

$$
\mathbf{u}=\left[\begin{array}{lll}
p_{\text {gyro }} & q_{\text {gyro }} & r_{\text {gyro }}
\end{array}\right]^{\top},
$$

The nonlinear equations that compose the model are from an improved dynamic model developed in [10],

$$
\begin{gathered}
{\left[\begin{array}{c}
\dot{f}_{j} \\
\dot{s}_{j} \\
\dot{d}_{j}
\end{array}\right]=\mathbf{R}_{b}^{n_{j}}\left(\phi, \theta, \psi_{j}\right)\left[\begin{array}{c}
u \\
v \\
w
\end{array}\right],} \\
{\left[\begin{array}{c}
\dot{\phi} \\
\dot{\theta} \\
\dot{\psi}
\end{array}\right]=\left[\begin{array}{ccc}
1 & \sin \phi \tan \theta & \cos \phi \tan \theta \\
0 & \cos \phi & -\sin \phi \\
0 & \frac{\sin \phi}{\cos \theta} & \frac{\cos \phi}{\cos \theta}
\end{array}\right]\left[\begin{array}{c}
p \\
q \\
r
\end{array}\right],} \\
{\left[\begin{array}{c}
\dot{u} \\
\dot{v} \\
\dot{w}
\end{array}\right]=\left[\begin{array}{cc}
-g \sin \theta+(v r-w q)-\frac{F_{d_{x}}}{m_{F_{d_{y}}}} \\
g \sin \phi \cos \theta+(w p-u r)-\frac{F^{m}}{m} \\
g \cos \phi \cos \theta+(u q-v p)-\frac{T}{m}
\end{array}\right],} \\
{\left[\begin{array}{c}
\dot{\beta}_{x} \\
\dot{\beta}_{y} \\
\dot{\beta}_{z}
\end{array}\right]=\left[\begin{array}{l}
0 \\
0 \\
0
\end{array}\right] .}
\end{gathered}
$$

The rotation matrix in (3) from the body frame to the node $j$ frame $\mathbf{R}_{b}^{n_{j}}\left(\phi, \theta, \psi_{j}\right)$, is as defined in [11]. The rotation rates $p, q$ and $r$ are defined as the gyroscope measurement minus the bias, i.e. $p=p_{\text {gyro }}-\hat{\beta}_{x}$. The body drag forces $F_{d_{x}}$ and $F_{d_{y}}$ in (5) are linearized with respect to hover to be

$$
\begin{aligned}
& F_{d_{x}} \approx-\mu u, \\
& F_{d_{y}} \approx-\mu v,,
\end{aligned}
$$

where $\mu=6 \lambda_{1} \bar{\omega}, \bar{\omega}$ is the average motor speed at hover and $\lambda_{1}$ is an aerodynamic constant.

This improved model of the vehicle dynamics provides the ability to fully utilize the information contained in the IMU measurements. As a consequence, estimation accuracy improves and the requirements for view matching or any other exteroceptive measurement updates are reduced [12], [13].

\section{B. Estimation}

Due to the fast dynamics of the hexacopter platform, we require state estimates at a quick rate for the control to stabilize the platform. In contrast to our previous work [13], we are now using a single filter for all states.

The continuous time model, (3) through (6), is used to propagated the state forward in time. The filter covariance is propagated using numerical integration of the equation

$$
\dot{\mathbf{P}}=\mathbf{A P}+\mathbf{P A}^{T}+\gamma\left(\mathbf{B G B}^{T}+\mathbf{Q}\right)
$$

where $\mathbf{B}$ is the Jacobian of (3) through (6) with respect to the inputs (2) and $\gamma$ is a constant between zero and one. The diagonal matrix $\mathbf{G}$ is the measured covariance on the inputs (2). A is the Jacobian of (3) through (6) with respect to the filter states and $\mathbf{Q}$ is the diagonal, hand-tuned portion of the process uncertainty. Accounting for the input noise appropriately allows this filter to be tuned easily.

There are two measurement updates for the filter. The first uses accelerometers to update the body velocities. We estimate the body $b_{x}$ and $b_{y}$ axis accelerometer measurements from the state as

$$
\mathbf{h}_{a c c}=\left[\begin{array}{l}
-\frac{\mu}{m} u \\
-\frac{\mu}{m} v
\end{array}\right],
$$

which is used in the measurement update

$$
\begin{aligned}
\mathbf{L}_{a c c} & =\mathbf{P C}_{a c c}\left(\mathbf{R}_{a c c}+\mathbf{C}_{a c c} \mathbf{P} \mathbf{C}_{a c c}^{\top}\right)^{-1}, \\
\mathbf{P}^{+} & =\left(\mathbf{I}-\mathbf{L}_{a c c} \mathbf{C}_{a c c}\right) \mathbf{P}^{-}, \\
\mathbf{x}^{+} & =\mathbf{x}^{-}+\mathbf{L}_{a c c}\left(\mathbf{y}_{a c c}-\mathbf{h}_{a} c c\right) .
\end{aligned}
$$

The notation $Y^{-}$and $Y^{+}$describes the variable $Y$ before and after the measurement update. The Jacobian of $\mathbf{h}_{a c c}$ is $\mathbf{C}_{a c c}$. The covariance $\mathbf{R}_{a c c}$ quantifies the noise on the accelerometer measurements.

The second measurement update is completed when position and yaw information are received from the view matching algorithm. Since the position and yaw states are relative, the predicted measurement is simply the current position and yaw states

$$
\mathbf{h}_{\text {pos }}=\left[\begin{array}{c}
f_{j} \\
s_{j} \\
d_{j} \\
\psi_{j}
\end{array}\right] .
$$

The measurement update is completed in a manner similar to the accelerometer update, only the Jacobian $\mathbf{C}_{a c c}$, noise $\mathbf{R}_{a c c}$, and innovation $\mathbf{y}_{a c c}-\mathbf{h}_{a} c c$ are changed to represent the information for the view matching.

One challenge with the position and yaw measurement updates is that they are delayed. The delay is due to the required processing time and the transmission time when the computation is performed off-board. Applying the measurement update requires that the state and covariance be brought back to the image time, the measurement update be calculated and the state and covariance then be repropagated forward using IMU measurements until the current time is again reached. The state, covariance and IMU information are saved at each timestep to accommodate this requirement.

When a new node is created, the relative portions of the 
state and covariance must change. The new node states must be augmented in the filter, with the mean values initialized to zero. The old node states are marginalized out after creating the edge between the old and new nodes.

\section{Map}

The map used in this work is a collection of nodes and edges in a graph framework, illustrated in Figure 1. The nodes in the graph represent the 3D position and heading of the vehicle when the keyframes are saved. Edges in the graph represent the estimated transformations between nodes. The only edges considered in this work come from the view matching, through the EKF. Other edges could feasibly be included, such as those created by visual recognition loop closures.

Using only odometry constraints, the map will gradually drift and the location estimates of the nodes will not be consistent with global truth. Loop closure constraints are essential for maintaining a globally consistent map. However, as explained above, the approach outlined here can work with either scenario.

\section{View Matching}

View matching is the process of estimating the $6 \mathrm{DOF}$ change in pose between a current image and a reference image. We have written our own view matching algorithm that uses a Kinect as the exteroceptive sensor. The algorithm estimates the pose changes between the current node keyframe and the current image, to update the relative states of the EKF. We include a short summary of the algorithm here.

FAST features and BRIEF descriptors are extracted from a gray version of the current image. The image is binned to allow an even dispersion of the features across the image. Next, correspondence between the current image features and the keyframe features are estimated using a $\mathrm{k}^{\text {th }}$ nearestneighbor (KNN) search from OpenCV. RANSAC is then employed to find a pose motion estimate, in which we use the three point singular value decomposition (SVD) algorithm [14]. The keyframe features are projected onto the current image frame to find inlier matches. Feature positions must match within one pixel for the pair to be declared an inlier. The inliers from the best motion estimate are used to create a least-squares estimate using the same SVD algorithm[14].

Since this information will be used in a measurement update in the EKF, an estimate of the measurement covariance $R_{v o}$ is necessary. We adapt the method outlined by [6].The motion estimate is sent back to the EKF as a measurement update along with the covariance.

The view matching algorithm is not yet sufficiently mature for in-the-loop testing. We currently approximate the view matching data using help from our motion capture system for the in-the-loop results. We synthesize view matching measurements by

1) down-sampling motion capture data to a $4 \mathrm{~Hz}$ update rate,
2) make the motion capture data relative and transform it into the current camera frame,

3) adding a stochastic delay representative of sending images offline for processing, and

4) adding zero-mean Gaussian noise with a standard deviation of $0.02 \mathrm{~m}$ in each direction.

We acknowledge that there are other artifacts of view matching that may not be modeled by these conditions, such as motion blur and false correspondence, yet we believe that these approximations provide a sufficient level of realism to examine the performance of the estimation and control.

\section{E. Control}

The control framework is based on previous work [9], where we describe a change of inputs to express the nonlinear equations (5) linearly.We only use a feedforward input to counter gravity.

Figure 2 illustrates the control in the system diagram. The estimator estimates the control states $\hat{\mathbf{x}}_{c}$, which are compared to the desired states $\mathbf{x}_{d}$. The error states $\tilde{\mathbf{x}}_{c}$ are used by the LQR control law to supply the linear inputs $\mathbf{u}_{c}$, to which the acceleration of gravity is subtracted. The linear control inputs are then converted to the nonlinear inputs $\nu_{c}$ expected by the hexacopter.

The states for the control are

$$
\mathbf{x}_{c}=\left[\begin{array}{lllllll}
f_{j} & s_{j} & d_{j} & \dot{f}_{j} & \dot{s}_{j} & \dot{d}_{j} & \psi_{j}
\end{array}\right]^{T},
$$

where the body frame velocities $u, v$ and $w$ in (1) are converted to node-frame velocities using $\mathbf{R}_{b}^{n_{j}}\left(\phi, \theta, \psi_{j}\right)$. The linear input for the control, $\mathbf{u}_{c}$, is

$$
\mathbf{u}_{c}=\left[\begin{array}{llll}
\ddot{f}_{j_{c}} & \ddot{s}_{j_{c}} & \ddot{d}_{j_{c}} & \dot{\psi}_{j_{c}}
\end{array}\right]^{\top} .
$$

Through the change of variables detailed in [9], the dynamics can be expressed in state-space form. We are able to use the same linear quadratic regulator (LQR) control law. After the LQR control law is applied, the acceleration due to gravity is subtracted from the $\ddot{d}_{j_{c}}$ component of $\mathbf{u}_{c}$.

The nonlinear control input $\nu_{c}$ is

$$
\nu_{c}=\left[\begin{array}{llll}
T_{c} & \phi_{c} & \theta_{c} & r_{c}
\end{array}\right]^{\top}
$$

where $T_{c}$ is the commanded thrust, $\phi_{c}$ is the commanded roll angle, $\theta_{c}$ is the commanded pitch, and $r_{c}$ is the commanded body-frame yaw rate. These inputs are calculated from $\mathbf{u}_{c}$ through $f^{-1}$ in Figure 2, which is described by

$$
\begin{aligned}
T_{c} & =\gamma_{T} m \sqrt{u_{c_{1}}^{2}+u_{c_{2}}^{2}+u_{c_{3}}^{2}}, \\
r_{c} & =\gamma_{r}\left(\dot{\psi}_{j} \cos \theta \cos \phi-\dot{\theta} \sin \phi\right), \\
\phi_{c} & =\gamma_{\phi} \sin ^{-1}\left(-z_{2}\right), \\
\theta_{c} & =\gamma_{\theta} \tan ^{-1}\left(\frac{z_{1}}{z_{3}}\right),
\end{aligned}
$$

where

$$
\left[\begin{array}{l}
z_{1} \\
z_{2} \\
z_{3}
\end{array}\right]=\mathbf{R}\left(\psi_{j}\right) \mathbf{u}_{c} \frac{m}{-T_{c}} .
$$


The subscript number denotes an element in a vector, i.e. $u_{c_{1}}$ is the first element in the vector $\mathbf{u}_{c}$. The mass of the vehicle is $m$. The constants $\gamma_{T}, \gamma_{r}, \gamma_{\phi}$ and $\gamma_{\theta}$ are used to convert the control inputs into the required format for the hexacopter. The rotation matrix $\mathbf{R}\left(\psi_{j}\right)$ is a right-handed rotation about the $d_{j}$ axis by the yaw angle $\psi_{j}$.

The path manager is modeled after the algorithms outlined in [11][Ch. 10 and 11]. The input to the algorithm is a list of waypoints for the vehicle to follow. The vehicle follows the waypoints in order by facing towards the next waypoint and moving at a constant velocity. Since the states are relative and the frame of reference changes throughout the flight, the waypoints are expressed in the new node frame each time a change in nodes occurs.

\section{RESULTS}

The results for two scenarios are presented. The first are results of delayed view matching estimates incorporated in the EKF, using recorded information. Kinect imagery and IMU data were recorded from a flight and used to create estimates offline. The second set of results are produced online with estimates in the control loop, where view matching data is synthesized as explained above.

\section{A. Offline Estimation Results Using Visual Data}

Figures 3 and 4 show a window of the performance of the estimation approach combined with actual view matching, using images recorded at $5 \mathrm{~Hz}$. The estimator runs at the rate IMU information is received, $40 \mathrm{~Hz}$. The rotations and translations from the view matching algorithm were delayed by an average of 0.43 seconds before they were applied to the filter. Truth information from the motion capture system has been made relative for purposes of the comparison. The times at which new nodes are declared are marked on the figures.

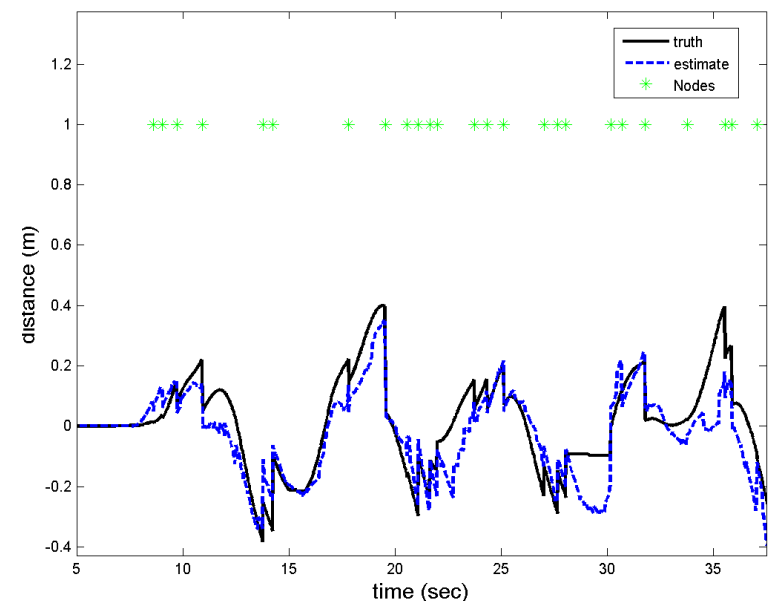

Fig. 3. Relative forward position $f$ truth and estimates. This data is produced using only the IMU and view matching algorithms from data recorded during a manually-controlled flight. The image data was recorded at $5 \mathrm{~Hz}$, with an average delay of 0.43 seconds (and a standard deviation of 0.27 seconds). The star data points represent each time a new node was declared.

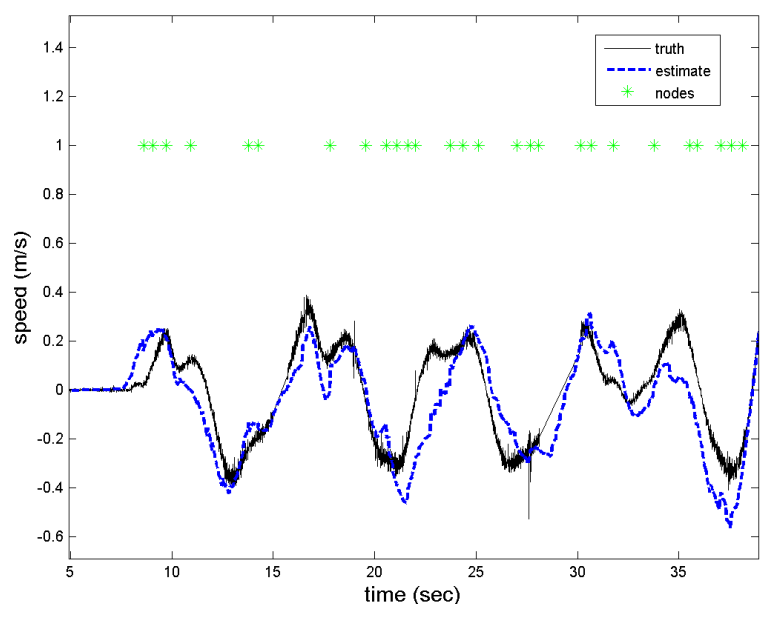

Fig. 4. Body frame velocity $u$ truth and estimates. This data is produced using only the IMU and view matching algorithms from data recorded during a manually-controlled flight. Notice that there are no jumps in the continuity, as the body-frame velocity is not relative.

When a new node is created the truth and estimates jump to a new position because the data presented in Figure 3 is relative to the current node. The jumps are not always to zero because the data are delayed.There is a section of bad truth data when time ranges between about 28 and 30 seconds.

\section{B. Flight Results}

A few flight results are shown below, all with the estimates in the control loop. All the results shown also use the delayed image data at $4 \mathrm{~Hz}$. The filter and control algorithms run at about $40 \mathrm{~Hz}$, which is the rate at which the IMU and altimeter data are received. The estimation and control are run on an Intel Atom board computer that is mounted to the hexacopter. The only data that is received from the ground station is the wirelessly-transmitted motion capture data used to simulate vision, as mentioned above. Figures 5 and 6 show examples

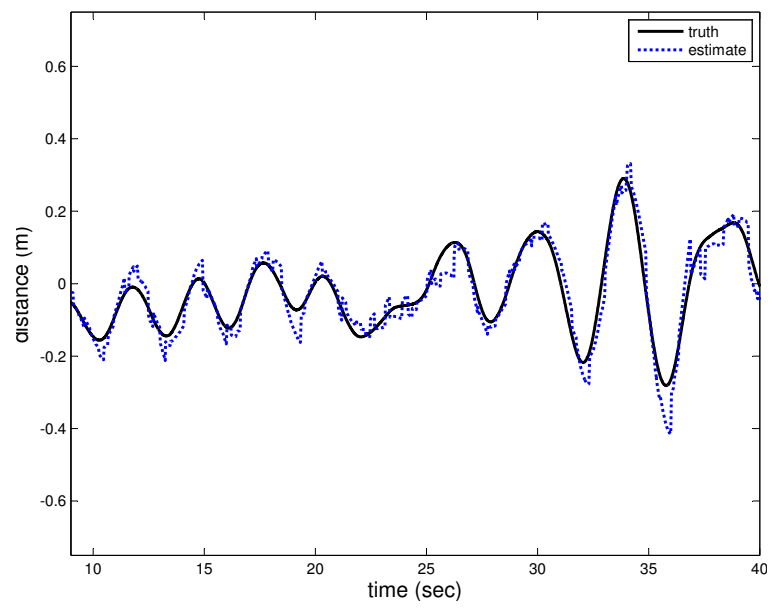

Fig. 5. Inertial north truth and estimates. Image-like data submitted at 4 $\mathrm{Hz}$, delayed. Estimates in the control loop.

of the typical performance of the estimates obtained by the 


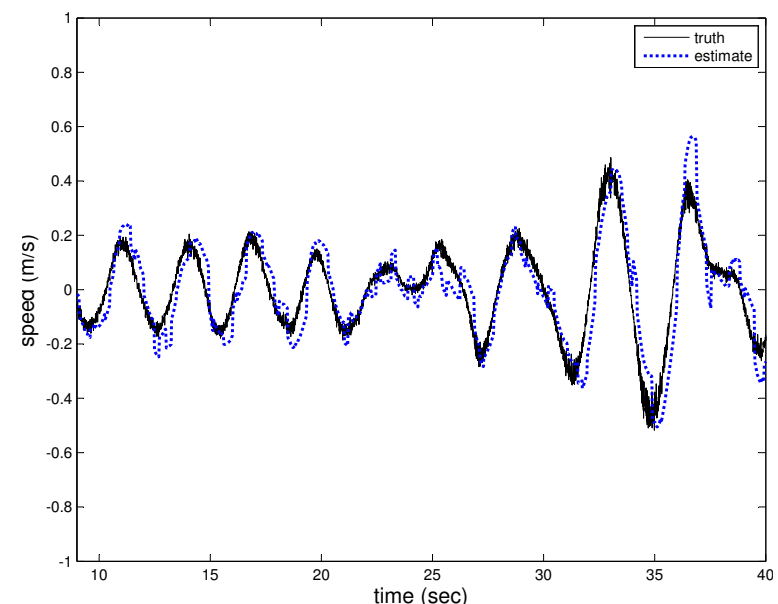

Fig. 6. Body frame velocity $u$ truth and estimates. Image-like data submitted at $4 \mathrm{~Hz}$, delayed. Estimates in the control loop.

filter compared to motion capture truth. This flight was an autonomous hover about the inertial point $(0,0,-0.6)$. Notice that the edges between each node have been summed to provide the inertial-frame estimates for ease in comparing the performance, however the filter was calculating the relative, node-based states for the duration of the flight.

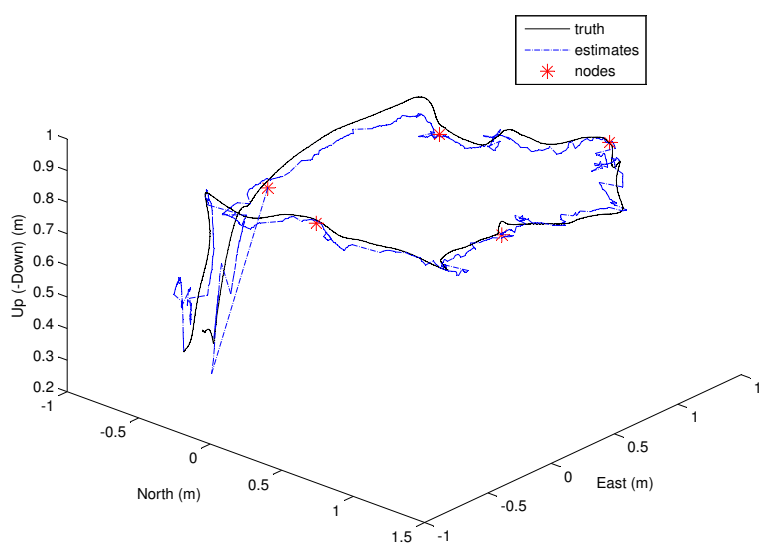

Fig. 7. 3D path of the hexacopter, with truth and estimates. Points represent nodes. Image-like data is submitted at $4 \mathrm{~Hz}$ and is delayed. Estimates are in the control loop.

Figure 7 shows a 3D path of the hexacopter as it navigates around a rectangle. Again, the global estimates along with the global truth are shown. The nodes that were automatically instantiated are shown in their 3D positions. The hexacopter smoothly transitions between each node, without jumps in the estimates nor with any pauses in motion. This is due to handling the augmentation and marginalizing of the state correctly.

\section{CONClusions And Future Work}

A relative, vision-based framework, like the approach described here, is an important step in furthering the capabilities of indoor aerial navigation. Current implementations that require global navigation often suffer deficiencies such as the need for additional states to incorporate measurements, waiting periods to process global consistency, and schemes to accommodate large jumps in pose when loop closures occur.

We have detailed the efforts to this point on creating a platform to provide a relative navigation capability. The navigation based on the EKF is robust, providing quality estimates despite delayed, low update-rate vision information. This facilitates an onboard implementation. The control, although basic, provides the capability to follow waypoint paths in the relative frame.

Foremost on the list of future work is the completion and integration of the view matching algorithm. Our vision algorithm should provide updates for in-the-loop experiments as it matures to the level of similar algorithms.

\section{ACKNOWLEDGMENTS}

This work is supported through the DoD SMART Scholarship program.

\section{REFERENCES}

[1] A. Bachrach, S. Prentice, R. He, and N. Roy, "Range: Robust autonomous navigation in gps-denied environments," Journal of Field Robotics, vol. 28, no. 5, pp. 644-666, 2011.

[2] G. Chowdhary, J. D. Michael Sobers, C. Pravitra, C. Christmann, A. Wu, H. Hashimoto, C. Ong, R. Kalghatg, and E. N. Johnson, "Integrated guidance navigation and control for a fully autonomous indoor uas," in Guidance Navigation and Control. AIAA, 2011.

[3] S. Ahrens, D. Levine, G. Andrews, and J. P. How, "Vision-based guidance and control of a hovering vehicle in unknown, GPS-denied environments," in Proc. IEEE Int. Conf. Robotics and Automation, 2009, pp. 2643-2648.

[4] M. Blosch, S. Weiss, D. Scaramuzza, and R. Siegwart, "Vision based MAV navigation in unknown and unstructured environments," in Proc. IEEE Int Robotics and Automation (ICRA) Conf, 2010, pp. 21-28.

[5] L. Garcia Carrillo, A. Dzul Lpez, R. Lozano, and C. Pgard, "Combining stereo vision and inertial navigation system for a quad-rotor uav," Journal of Intelligent \& Robotic Systems, pp. 1-15.

[6] R. Voigt, J. Nikolic, C. Hurzeler, S. Weiss, L. Kneip, and R. Siegwart, "Robust embedded egomotion estimation," in Proc. IEEE/RSJ Int Intelligent Robots and Systems (IROS) Conf, 2011, pp. 2694-2699.

[7] K. Konolige, J. Bowman, J. Chen, P. Mihelich, M. Calonder, V. Lepetit, and P. Fua, "View-based maps," The International Journal of Robotics Research, vol. 29, no. 8, pp. 941-957, 2010.

[8] G. Grisetti, C. Stachniss, S. Grzonka, and W. Burgard, "A tree parameterization for efficiently computing maximum likelihood maps using gradient descent," in Proc. of robotics: science and systems, 2007.

[9] J. Ferrin, R. Leishman, R. Beard, and T. McLain, "Differential flatness based control of a rotorcraft for aggressive maneuvers," in Proc. IEEE Int. Conf. Intelligent Robots and Systems, 2011.

[10] P. Martin and E. Salaun, "The true role of accelerometer feedback in quadrotor control," in Proc. IEEE Int Robotics and Automation (ICRA) Conf, 2010, pp. 1623-1629.

[11] R. Beard and T. McLain, Small Unmanned Aircraft. Princeton University Press, 2012.

[12] R. Leishman, J. Macdonald, T. McLain, and R. Beard, "Accelerometers \& observers: Improving quadrotor state estimation," Robotics and Automation Magazine, September 2012, submitted.

[13] R. Leishman, J. Macdonald, S. Quebe, J. Ferrin, T. McLain, and R. Beard, "Using an improved rotorcraft dynamic model in state estimation," in Proc. IEEE Int. Intelligent Robots and Systems, San Fransisco, September 2011.

[14] K. S. Arun, T. S. Huang, and S. D. Blostein, "Least-squares fitting of two 3-d point sets," IEEE Trans. Pattern Anal. Mach. Intell., vol. 9, pp. 698-700, May 1987. 